\title{
The Effects of Training and Detraining on Metabolic Hormones
}

\section{in Rats}

\author{
Jae Hoon Jang, Chang Hwa Joo \\ Department of Football Science, Ho Nam University, Gwangju, Korea
}

PURPOSE: We conclude that exercise training-induced effects in metabolic hormones continue for a prolonged period of detraining, but they are not associated with exercise intensity or detraining-induced increases in body weight.The purpose of this study was to examine the effect of diverse exercise training intensities, training periods and detraining periods on metabolic hormones.

METHODS: Eighty-four male Sprague-Dawley rats (aged 6 weeks, 173-200 g) were divided into 12 different groups by training, detraining, and control conditions. Exercise training consisted of low-intensity training and high-intensity training programs performed in the laboratory on a motor-driven rodent treadmill once per day, 5 days per week for 12 weeks.

RESULTS: Body weight significantly increased in all the conditions after the 12-week training period, with significant interaction among the conditions $(p<.01)$. Body weights in the low-intensity trained detrained (LTD) and high-intensity trained detrained (HTD) groups were similar to those in the control groups after 6 weeks and 12 weeks of detraining $(p<.05)$. There were significant differences in the hormones (leptin, insulin, and cortisol) between the control and trained detrained condition after 12 weeks of training, and 6 weeks and 12 weeks of detraining $(p<.01)$, with no significant interaction between the trained detrained conditions $(p<.05)$.

CONCLUSIONS: We conclude that exercise training-induced effects in metabolic hormones continue for a prolonged period of detraining, but they are not associated with exercise intensity or detraining-induced increases in body weight.

Key words: Exercise training, Metabolism, Hormones

\section{INTRODUCTION}

Physical exercise, which represents the most variable fraction of energy expenditure, is the best way to treat obesity without side effects. Many animal [1-4] and human [5-7] studies have been conducted to investigate the effects of exercise and endurance training on leptin. For example, in rats, a single bout of swimming decreased plasma leptin by $30 \%$ and improved hypothalamic leptin signaling [2,3]. Abbenhardt et al. [6] reported that, in humans, chronic endurance exercise consisting of 45 minutes of moderate-to-vigorous intensity exercise 5 days per week for 12 months decreased leptin concentrations by $30 \%$ with a reduction in body weight. These results suggest that acute and chronic exercise may improve hypothalamic leptin sensitivity in obesity.

Detraining refers to the partial or complete loss of training adaptation due to a reduction or cessation of regular or vigorous fitness training [8]. The main factor associated with detraining is the reduction of physical fitness. Several studies have shown that detraining causes a decrease in exercise performance in untrained $[8,9]$ and trained individuals $[10,11]$. From a metabolic viewpoint, detraining can cause an increase in adiposity, insulin resistance, and adipokines secretion, which may result in the development of chronic metabolic diseases [12,13].

Most studies examining metabolic hormones in response to physical training have only included a few weeks of training and detraining $(\sim 8$ weeks) [14-16]. It is unclear whether a training-induced change in metabolic hormones occurs after a prolonged period of detraining. To our knowledge, few studies have investigated metabolic hormones in response to a prolonged period of detraining ( 12 weeks) after low-intensity and high-intensity exercise training in rats. It is possible that a more

Corresponding author: Chang Hwa Joo Tel +82-62-940-3602 Fax +82-62-940-3741 E-mail footballerlahonam.ac.kr

Received 15 Feb 2017 Revised 13 Mar 2017 Accepted 5 Apr 2017

(a) This is an Open Access article distributed under the terms of the Creative Commons Attribution Non-Commercial License (http://creativecommons.org/licenses/by-nc/4.0/) which permits unrestricted non-commercial use, distribution, and reproduction in any medium, provided the original work is properly cited. 
controlled methodological approach using 12 groups (3 conditions control, low-intensity, and high-intensity) $\times 4$ time points [pre-training, posttraining, and 6-weeks and 12-weeks post-detraining] can accurately examine the effect of the exercise training response to different exercise intensities, i.e., a short and prolonged period of detraining on metabolic hormones. The aim of this study, therefore, was to determine the effect of different exercise training intensities and detraining periods on metabolic hormones.

\section{MATERIALS AND METHODS}

\section{Animals}

Eighty-four male Sprague-Dawley rats aged 6 weeks (173-200 g) were obtained from Orient Bio (Seongnam, Korea) and housed in cages under automatically controlled environmental conditions of temperature $(22 \pm$ $\left.1^{\circ} \mathrm{C}\right)$, humidity $(55 \pm 5 \%)$, and lighting (12:12-hour light-dark cycle) with standard rodent chow (5.8\% fat, Orient Bio) and water ad libitum at the Physiology Department Animal Facility, Kangwon National University. Rats were cared for per the university established Guidelines for Animal Experiments.

Rats were randomly assigned to 1 of 12 groups: sedentary controls $(\mathrm{n}=7)$, low-intensity trained controls $(\mathrm{n}=7)$, high-intensity trained controls $(n=7)$, sedentary controls for 12 weeks $(n=7)$, low-intensity trained for 12 weeks $(n=7)$, high-intensity trained for 12 weeks $(n=7)$, sedentary controls for 18 weeks $(n=7)$, low-intensity trained detrained for 6 weeks $(n=7)$, high-intensity trained detrained for 6 weeks $(n=7)$, sedentary controls for 24 weeks $(n=7)$, low-intensity trained detrained for 12 weeks $(\mathrm{n}=7)$, and high-intensity trained detrained for 12 weeks $(\mathrm{n}=7)$.

\section{Exercise training and detraining}

Low-intensity training $\left(20 \mathrm{~m} / \mathrm{min} ; 60 \% \mathrm{VO}_{2} \mathrm{max}\right)$ and high-intensity training (28 m/min; 75-80\% $\mathrm{VO}_{2} \mathrm{max}$ ) groups performed a 1,000-m run on a motor-driven rodent treadmill once per day, 5 days per week for 12 weeks [17]. Rats were adapted to the exercise protocol for 1 week before beginning the exercise training protocol. Detrained rats underwent training as described previously, and then they remained sedentary for 6 or 12 weeks. Rats were allowed to roam their cages freely during detraining.

\section{Plasma analysis}

All rats were fasted for 12 hours after 40 hours from the final bout of exercise. They were anesthetized with diethyl ether (Wako Pure Chemi- cal Industries, Ltd., Osaka, Japan), and a blood sample was taken from the abdominal artery $(10 \mathrm{~mL})$ for analysis. Samples were centrifuged $\left(3,000 \mathrm{rpm}\right.$ for 15 minutes at $\left.4^{\circ} \mathrm{C}\right)$ to obtain serum, which was stored at $-80^{\circ} \mathrm{C}$ for post-exercise analysis of leptin, insulin, and cortisol levels Leptin and insulin levels were measured with a specific radioimmunoassay kit (Linco Research, St. Charles, MO, USA). The cortisol level was analyzed enzymatically using an autoanalyzer (Hitachi 747, Hitachi, Tokyo, Japan).

\section{Statistical analysis}

All data are presented as a mean \pm standard deviation. Two-way repeated-measures analysis of variance was used to determine the main effect of time and/or detraining on study outcomes (body weight, leptin, insulin, and cortisol levels). Tukey post-hoc tests were performed when a significant main effect or interaction was obtained to determine differences between means. Statistical significance was set at $p<.05$ (two-tailed).

\section{RESULTS}

\section{Body weight}

Changes in body weight are presented in Fig. 1. The exercise-trained groups had a lower body weight after 12 weeks of training than the control groups $(p<.01)$. Body weight increased after 6 weeks and 12 weeks of detraining in all conditions. Body weights in the trained and detrained

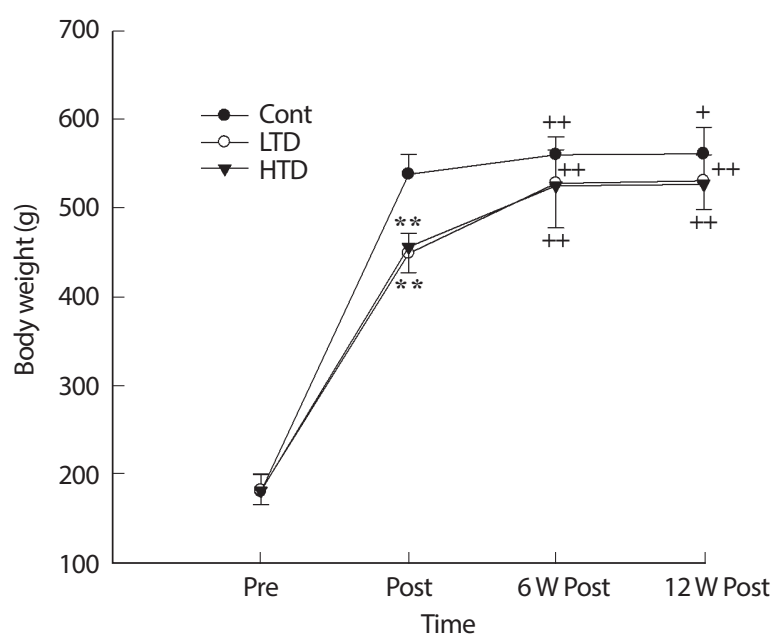

Fig. 1. Body weight before exercise training (Pre), after $12 \mathrm{~W}$ exercise training (Post), $6 \mathrm{~W}$ and $12 \mathrm{~W}$ of training cessation (6 W Post, $12 \mathrm{~W}$ Post). Cont, control; LTD, low intensity training; HTD, high intensity training. **Significant difference $(p<.01)$ between Cont and exercise training groups (LTD [upper] and HTD [lower]), ${ }^{+} p<.05 ;{ }^{++} p<.01$ Significant difference from Post in the control, LTD and HTD. 


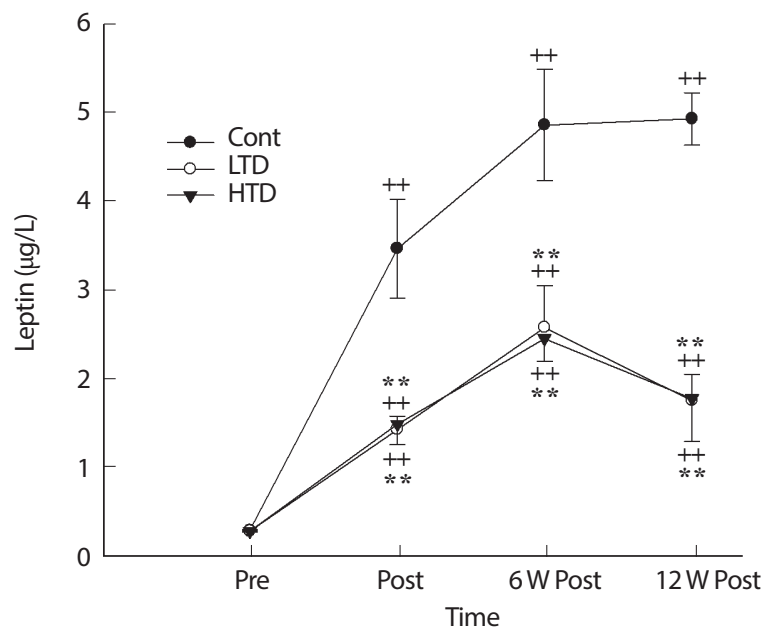

Fig. 2. Leptin concentration before exercise training (Pre), after $12 \mathrm{~W}$ exercise training (Post), $6 \mathrm{~W}$ and $12 \mathrm{~W}$ of training cessation ( $6 \mathrm{~W}$ Post, $12 \mathrm{~W}$ Post). Cont, control; LTD, low intensity training; HTD, high intensity training. **Significant difference $(p<.01)$ between Cont and exercise training groups (LTD [upper] and HTD [lower]), ${ }^{++}$Significant difference $(p<.01)$ from Pre in the control, LTD and HTD.

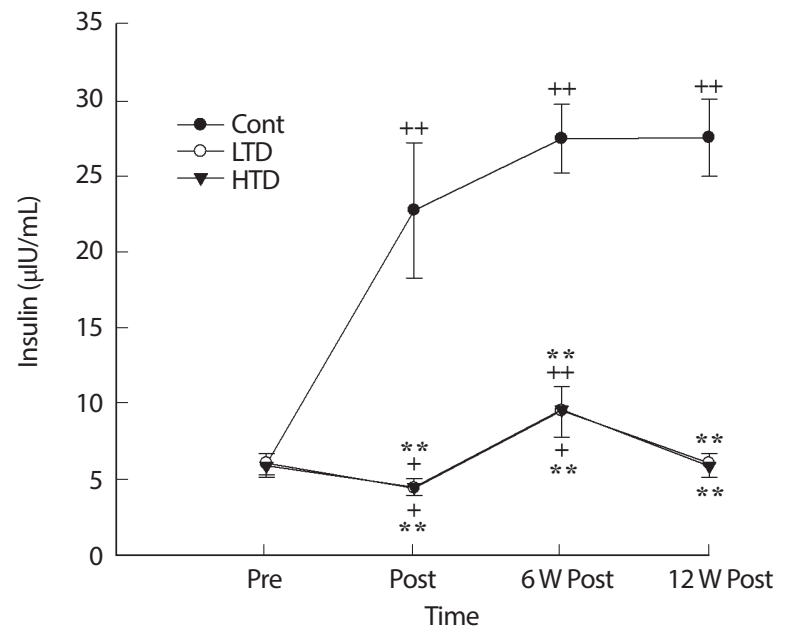

Fig. 3. Insulin concentration before exercise training (Pre), after $12 \mathrm{~W}$ exercise training (Post), $6 \mathrm{~W}$ and $12 \mathrm{~W}$ of training cessation ( $6 \mathrm{~W}$ Post, $12 \mathrm{~W}$ Post). Cont, control; LTD, low intensity training; HTD, high intensity training. **Significant difference $(p<.01)$ between Cont and exercise training groups (LTD [upper] and HTD [lower]), Significant difference $\left({ }^{+} p<.05,{ }^{++} p<.01\right)$ from Pre in the control, LTD and HTD.

groups were similar to those in the control groups $(p>.05)$.

\section{Plasma leptin level}

The plasma leptin level increased significantly after 6 weeks of detraining in all conditions with significant differences between the control and trained detrained groups ( $p<.01$; Fig. 2). After 12 weeks of detraining, the trained detrained groups exhibited a decrease in leptin levels $(p>.05)$.

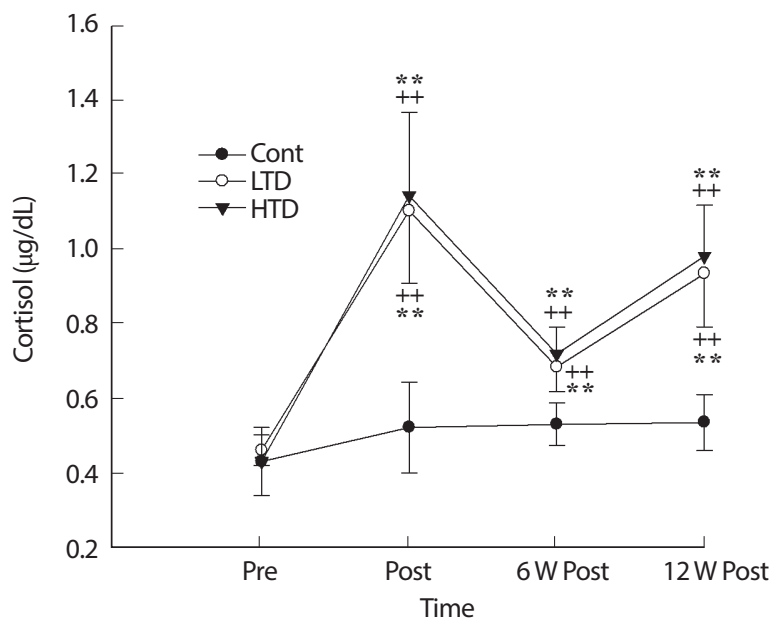

Fig. 4. Cortisol concentration before exercise training (Pre), after $12 \mathrm{~W}$ exercise training (Post), $6 \mathrm{~W}$ and $12 \mathrm{~W}$ of training cessation ( $6 \mathrm{~W}$ Post, $12 \mathrm{~W}$ Post). Cont, control; LTD, low intensity training; HTD, high intensity training. **Significant difference $(p<.01)$ between Cont and exercise training groups (LTD [lower] and HTD [upper]), ${ }^{++}$Significant difference $(p<.01)$ from Pre in the control, LTD and HTD.

\section{Plasma insulin level}

The plasma insulin level increased gradually during the 24-week experimental period in the control groups, with significant differences in the trained detrained groups $(p<.01$; Fig. 3). There were no significant differences between the LTD and HTD groups during the experimental period $(p>.05)$.

\section{Plasma cortisol level}

Compared with the control groups, both detraining treatments resulted in a higher concentration of cortisol at all points during the test $(p<.01$; Fig. 4). The change in cortisol between the LTD and HTD groups was similar during the experimental period; however, differences between the groups were not significant $(p>.05)$.

\section{DISCUSSION}

The purpose of this study was to examine the effect of various training intensities, training periods, and detraining periods on metabolic hormones. We found that 1) endurance training resulted in lower leptin and insulin levels compared to no training; 2) training-induced lower leptin and insulin levels were significantly different compared to the no training condition after 6 and 12 weeks of detraining; and 3) changes in leptin and insulin levels due to endurance training are intensity-independent. 
Leptin plays an essential role in the regulation of food intake and energy balance [5]. Most previous studies have examined the effects of exercise training on serum leptin concentrations $[5,18,19]$, but few studies have investigated the effect of prolonged periods of detraining on leptin levels. For example, leptin levels decreased significantly following a 20week period of endurance training [5] and a 6-month resistance training program [20]. In line with previous observations, we found that 12 weeks of endurance training resulted in significant differences in leptin levels between training and control conditions, and leptin levels remained lower in training conditions compared to controls at 6 and 12 weeks of detraining. However, leptin levels were not different between exercise intensity conditions for the 24 -week experimental period. This is inconsistent with previous findings, which indicated that absolute leptin levels and the percent decrease from baseline were higher in highintensity training compared with other groups (control, low-intensity training, and moderate-intensity training) after 6 months of training and 6 months of detraining [20]. The reason for the discrepancy between our findings and those of Fatouros et al. [20] is unclear, but it may be due to the exercise modality (continuous exercise on a treadmill vs. resistance exercise). Our findings suggest that the exercise duration should be considered rather than the exercise intensity for obesity treatment to mediate energy balance and body weight through leptin responses to continuous aerobic exercise.

In the present study, the insulin concentration was significantly increased in the control groups. This response can be directly associated with the degree of weight gain [13]. However, insulin decreased after 12 weeks of high-intensity and low-intensity training in the current study. This is in line with previous studies showing that low-volume/moderate intensity ( 12 miles walking/wk at 40-55\% $\mathrm{VO}_{2}$ peak) and high-volume/ high intensity ( 20 miles jogging/wk at $65-80 \% \mathrm{VO}_{2}$ peak) exercises for 6 months significantly decreased the insulin level, with significant decrease in body weight [21]. Interestingly, in the current study, the insulin level was significantly lower in both training conditions after 6 and 12 weeks of detraining, even though body weight matched the controls simultaneously. The concentration of serum insulin, therefore, may not depend on large changes in body weight after a prolonged period detraining. Our findings thus align with other observations that traininginduced increases in insulin action continued for 6 and 15 days of detraining in overweight subjects [22,23], and the training-induced improvement in insulin sensitivity was not associated with changes in body weight, body fat, and adiponectin [24]. Nassis et al. [24] suggested that enhanced insulin sensitivity may be due to an increase in the glucose transporter-4 concentration in skeletal muscle and training-induced increase in skeletal muscle mass, which is the primary target tissue for insulin action. Together, these observations suggest that adaptations, such as changes in the ability of muscles to metabolize glucose [25], associated with moderate-intensity exercise and high-intensity exercise training for 12 weeks, may improve insulin action that remains for prolonged periods of detraining.

There were significant differences in the cortisol level between the control and exercise training groups throughout the testing periods in the present study. The cortisol level was higher in both training groups than in the controls. These increases are likely the result of enhanced stimulus to the hypothalamic-pituitary-adrenocortical axis [26]. However, for reasons that are unclear, cortisol levels were not significantly different between training groups throughout the experimental period. Differences in exercise duration can explain the lack of differences between the training groups. Indeed, Hill et al. [27] reported that the circulating cortisol level was greater in a high-intensity exercise group (80\% $\left.\mathrm{VO}_{2} \max \right)$ than in a low-intensity exercise group $\left(60 \% \mathrm{VO}_{2} \mathrm{max}\right)$ for $\mathrm{du}-$ rations of 30 minutes. Therefore, the training-induced increase in the cortisol level may improve cortisol-dependent metabolism (e.g., stimulating gluconeogenesis in the liver), which persists over a prolonged time of detraining.

There were several limitations to the current study, including the analysis of body tissues, such as the brain, muscle, and fat. Unfortunately, we could not analyze tissues to determine cellular and molecular responses to exercise training and a prolonged period of detraining. Exercise training and detraining may differently affect changes in cell signaling, which affects the release of metabolic hormones and the expression of hormones' receptors. Further research is needed to investigate the effects of exercise training and detraining on cell signaling.

In conclusion, our findings indicate that training-induced changes in leptin, insulin, and cortisol concentrations persist for at least 12 weeks of detraining. In contrast, exercise intensity is not important for mediating changes in circulating hormone concentrations after training and detraining. These findings suggest that low-intensity exercise $\left(60 \% \mathrm{VO}_{2} \mathrm{max}\right)$ training is sufficient enough to affect the endocrine system.

\section{REFERENCES}

1. Zheng D, Wooter MH, Zhou Q, Dohm GL. The effect of exercise on 
ob gene expression. Biochemical and Biophysical Research Communications 1996;225(3):747-750.

2. Pagano C, Marzolo M, Granzotto M, Ricquier D, Federspil G, et al. Acute effects of exercise on circulating leptin in lean and genetically obese fa/fa rats. Biochemical and Biophysical Research Communications 1999;255(3):698-702.

3. Flores MB, Fernandes MF, Ropelle ER, Faria MC, Ueno M, et al. Exercise improves insulin and leptin sensitivity in hypothalamus of Wistar rats. Diabetes 2006;55(9):2554-2561.

4. Borg ML, Andrews ZB, Watt MJ. Exercise training does not enhance hypothalamic responsiveness to leptin or ghrelin in male mice. Journal of Neuroendocrinology 2014;26(2):68-79.

5. Perusse L, Collier G, Gagnon J, Leon AS, Rao DC, et al. Acute and chronic effects of exercise on leptin levels in humans. Journal of Applied Physiology 1997;83(1):5-10.

6. Abbenhardt C, McTiernan A, Alfano CM, Wener MH, Campbell KL, et al. Effects of individual and combined dietary weight loss and exercise interventions in postmenopausal women on adiponectin and leptin levels. Journal of Internal Medicine 2013;274(2):163-175.

7. Schild M, Eichner G, Beiter T, Zugel M, Krumholz-Wagner I, et al. Effects of Acute Endurance Exercise on Plasma Protein Profiles of Endurance-Trained and Untrained Individuals over Time. Mediators Inflamm 2016;2016:4851935.

8. Mujika I, Padilla S. Detraining: loss of training-induced physiological and performance adaptations. Part I: short term insufficient training stimulus. Sports Medicine 2000;30(2):79-87.

9. Kraemer WJ, Koziris LP, Ratamess NA, Hakkinen K, Triplett NT, et al. Detraining produces minimal changes in physical performance and hormonal variables in recreationally strength-trained men. The Journal of Strength \& Conditioning Research 2002;16(3):373-382.

10. Melchiorri G, Ronconi M, Triossi T, Viero V, De Sanctis D, et al. Detraining in young soccer players. The Journal of Sports Medicine and Physical Fitness 2014;54(1): 27-33.

11. Joo $\mathrm{CH}$. The effects of short-term detraining on exercise performance in soccer players. Journal of Exercise Rehabilitation 2016;12(1):54-59.

12. Booth FW, Laye MJ, Lees SJ, Rector RS, Thyfault JP. Reduced physical activity and risk of chronic disease: the biology behind the consequences. European Journal of Applied Physiology 2008;102(4):381-390.

13. Mazzucatto F, Higa TS, Fonseca-Alaniz MH, Evangelista FS. Reversal of metabolic adaptations induced by physical training after two weeks of physical detraining. International Journal of Clinical and Experi- mental Medicine 2014;7(8):2000-2008

14. Sertie RA, Andreotti S, Proenca AR, Campana AB, Lima-Salgado TM, et al. Cessation of physical exercise changes metabolism and modifies the adipocyte cellularity of the periepididymal white adipose tissue in rats. Journal of Applied Physiology 2013;115(3):394-402.

15. Kilic-Erkek O, Mergen-Dalyanoglu M, Kilic-Toprak E, Ozkan S, BorKucukatay M, et al. Exercise training and detraining process affects plasma adiponectin level in healthy and spontaneously hypertensive rats. Bratisl Lek Listy 2015;116(12):741-745.

16. Marschner RA, Pinto G, Borges J, Markoski MM, Schaan BD, et al. Short-term Detraining does not change insulin sensitivity and RBP4 in rodents previously submitted to aerobic exercise. Hormone and Metabolic Research 2017;49(1):58-63.

17. Kim DH, Kim SH, Kim WH, Moon CR. The effects of treadmill exercise on expression of UCP-2 of brown adipose tissue and TNF-alpha of soleus muscle in obese Zucker rats. Journal of Exercise Nutrition \& Biochemistry 2013;17(4):199-207.

18. Hopkins M, Gibbons C, Caudwell P, Webb DL, Hellstrom PM, et al. Fasting leptin is a metabolic determinant of food reward in over weight and obese individuals during chronic aerobic exercise training. International Journal of Endocrinology 2014;2014:323728.

19. Kim YS, Nam JS, Yeo DW, Kim KR, Suh SH, et al. The effects of aerobic exercise training on serum osteocalcin, adipocytokines and insulin resistance on obese young males. Clinical Endocrinology (Oxf) 2015; 82(5):686-694

20. Fatouros IG, Tournis S, Leontsini D, Jamurtas AZ, Sxina M, et al. Leptin and adiponectin responses in overweight inactive elderly following resistance training and detraining are intensity related. The Journal of Clinical Endocrinology 2005;90(11):5970-5977.

21. Houmard JA, Tanner CJ, Slentz CA, Duscha BD, McCartney JS, et al Effect of the volume and intensity of exercise training on insulin sensitivity. Journal of Applied Physiology 2004;96(1):101-106.

22. Dela F, Larsen JJ, Mikines KJ, Ploug T, Petersen LN, et al. Insulin-stimulated muscle glucose clearance in patients with NIDDM: Effects of one-legged physical training. Diabetes 1995;44(9):1010-1020.

23. Bajpeyi S, Tanner CJ, Slentz CA, Duscha BD, McCartney JS, et al. Effect of exercise intensity and volume on persistence of insulin sensitivity during training cessation. Journal of Applied of Physiology 2009; 106(4):1079-1085.

24. Nassis GP, Papantakou K, Skenderi K, Triandafillopoulou M, Kavouras SA, et al. Aerobic exercise training improves insulin sensitivity 
without changes in body weight, body fat, adiponectin, and inflammatory markers in overweight and obese girls. Metabolism 2005;54(11): 1472-1479.

25. Dengel DR, Galecki AT, Hagberg JM, Pratley RE. The independent and combined effects of weight loss and aerobic exercise on blood pressure and oral glucose tolerance in older men. American Journal of
Hypertension 1998;11(12):1405-1412.

26. Viru A, Viru M. Cortisol-essential adaptation hormone in exercise. International Journal of Sports Medicine 2004;25(6):461-464.

27. Hill E, Zack E, Battaglini C, Viru M, Viru A, et al. Exercise and circulating cortisol levels: the intensity threshold effect. Journal of Endocrinological Investigation 2008;31(7):587-591. 